\title{
Signaling Events in Axons and/or Dendrites Render Motor Neurons Vulnerable to Mutant Superoxide Dismutase Toxicity
}

\author{
Goo-Bo Jeong, ${ }^{1,2}$ Jelena Mojsilovic-Petrovic, ${ }^{1}$ Marco Boccitto, ${ }^{1}$ and Robert Kalb ${ }^{1}$ \\ ${ }^{1}$ Division of Neurology, Department of Pediatrics, Children's Hospital of Philadelphia, University of Pennsylvania School of Medicine, Philadelphia, \\ Pennsylvania 19104, and 2Department of Anatomy, Gachon University of Medicine and Science, Incheon 406-799, Korea
}

The survival of dorsal root ganglion and sympathetic neurons is promoted whether nerve growth factor (NGF) activates TrkA receptors on the cell body or the axon. Yet other aspects of neurotrophic factor actions (i.e., ability to promote axon growth, selection of neurochemical phenotype and engagement of signaling modules) differ as a function of the location of the ligand-receptor interaction. The extent to which these observations are relevant to CNS neurons is unknown. This may be particularly relevant to neurodegenerative diseases such as amyotrophic lateral sclerosis, where beneficial axon-target interactions are disturbed early in the disease process. Here we characterize the growth of pure motor neurons in compartment cultures and show that brain-derived neurotrophic factor (BDNF) stimulation of the cell body or axons/dendrites promotes survival. Expression of G37R mutant superoxide dismutase (SOD) in motor neurons will lead to death and this depends on BDNF activation of TrkB on axons and/or dendrites. BDNF action depends upon endocytosis of the BDNF-TrkB complex and de novo protein synthesis. These results highlight the importance of signaling events occurring in axons/dendrites in mutant SOD toxicity.

\section{Introduction}

The survival of developing neurons depends upon their ability to innervate appropriate target cells and obtain target-derived trophic support (Hamburger, 1958). Work using peripheral nervous system neurons has demonstrated that the nature of trophic factor signaling events differs as a function of location (Segal, 2003). For example, in dorsal root ganglion cells, extracellular-regulated kinase 5 (Erk5) conveys the prosurvival signal upon activation of TrkA receptors on axons. In contrast, Erk1/2 conveys the prosurvival signal upon activation of TrkA receptors on the cell body (Watson et al., 2001). In sympathetic neurons, phosphatidylinositol 3' kinase $\left(\mathrm{PI} 3^{\prime} \mathrm{K}\right)$ conveys the prosurvival signal upon activation of TrkA receptors on axons, while a non-PI3'K pathway conveys the prosurvival signal upon activation of TrkA receptors on the cell body (Kuruvilla et al., 2000). Thus, under normal circumstances, neurons must adjudicate multiple signaling events (those arising from the periphery vs those arising at the cell body) to maintain normal function. In addition, the specific signaling events may differ between distinct neuron types.

While BDNF promotes neuronal survival, it can also make neurons vulnerable to the toxicity of oxygen-glucose deprivation (Koh et al., 1995), oxidative stress (Kim et al., 2003), nitric oxide (Ishikawa et al., 2000), excitotoxicity (Fryer et al., 2000; Hu and

\footnotetext{
Received Sept. 14, 2010; revised 0ct. 28, 2010; accepted 0ct. 30, 2010.

This work was supported through the generosity of the Philadelphia Chapter of the ALS Association, the national office of the ALS Association, and the United States Public Health Service (NS 05325 and NS 064232). We thank Rachael Neve (Picower Center, MIT) for packaging recombinant HSVs used in these studies, Eran Perlson and Erika Holzbaur (University of Pennsylvania) for many important discussions as this project unfolded, and Rosalind Segal and laboratory for instructing us in the creation of compartment culture system.

Correspondence should be addressed to Dr. Robert Kalb, Children's Hospital of Philadelphia, Abramson Research Center, \#814, 3615 Civic Center Boulevard, Philadelphia, PA 19104. E-mail: kalb@email.chop.edu.

DOI:10.1523/JNEUROSCI.4824-10.2011

Copyright $\odot 2011$ the authors $\quad 0270-6474 / 11 / 310295-05 \$ 15.00 / 0$
}

Kalb, 2003), and proteotoxic stress (Mojsilovic-Petrovic et al., 2006). Here we show that this effect depends on BDNF stimulation of motor neuron neurites in a manner that involves ligandreceptor endocytosis and local protein synthesis. Our findings suggest that proteins synthesized in axon/dendrites in a BDNFdependent manner critically impact the ability of motor neurons to survive toxic insult.

\section{Materials and Methods}

Compartment culture. Thirty-five millimeter tissue culture dishes were coated with poly-D-lysine ( $50 \mu \mathrm{g} / \mathrm{ml}$, Sigma) and mouse laminin ( $5 \mu \mathrm{g} /$ $\mathrm{ml}$, Invitrogen) and a pin-rake was used to scratch parallel lines across the diameter of the dish. Next, a Teflon divider (Camp10, Tyler Research Instruments) seated with silicone grease (Dow Corning) was placed orthogonally across the scratch lines. The integrity of the seal was tested in every dish by placing sterile media in the neurite (side) compartments and looking for leakage into the cell body compartment after $24 \mathrm{~h}$ in the incubator. All dishes with leaks were discarded.

Pure motor neurons were obtained from E14 rat embryos as previously described (Fryer et al., 2000), and 40,000 purified motor neurons were plated in $30 \mu \mathrm{l}$ of media in the cell body chamber of the compartment culture (Campenot, 1977). It is difficult to determine precisely the total number of motor neurons that adhere to the substrate and survive because of the nonuniform distribution of cells in the cell body chamber, a variable degree of cell clumping, and the optical limitations imposed by the plastic dish.

"NB + CGC" media consists of Neurobasal media (Invitrogen) with 2\% horse serum, 5\% B27 (Invitrogen), 0.5 mm GlutaMax (Invitrogen), $50 \mu \mathrm{M} 2$-mercaptoethanol (Invitrogen), 1\% penicillin-streptomycin (Invitrogen), and $10 \mu \mathrm{M} 5$-fluoro-5'-deoxyuridine (Sigma) supplemented with $1 \mathrm{ng} / \mathrm{ml}$ of CT-1, GDNF, CNTF (Alomone Labs). Cells were maintained at $37^{\circ} \mathrm{C}$ with $5 \% \mathrm{CO}_{2}$. Media in the cell body compartment included $50 \mathrm{ng} / \mathrm{ml} \mathrm{BDNF}$ for the first $2 \mathrm{~d}$ and then changed to $1 \mathrm{ng} / \mathrm{ml}$ $\mathrm{BDNF}$ for the subsequent week. Media in the neurite compartment contained $50 \mathrm{ng} / \mathrm{ml} \mathrm{BDNF}$ for the first week of culture (to robustly promote 
neurite growth). Subsequently neurons were grown in NB + CGC until subsequent experimental manipulations.

CM-DiI labeling was accomplished by addition of chloromethyl DiI ( $1 \mu \mathrm{M}$, Invitrogen) to the neurite compartment overnight at $37^{\circ} \mathrm{C}$, then washing three times in culture media.

In toxicity studies, 14 DIV cultures were infected with $1 \mu$ l of recombinant herpes simplex virus (HSV) engineered to express G37R mutant SOD [viral titer was 3-5 $\times 10^{7}$ plaqueforming units $(\mathrm{PFU}) / \mathrm{ml}$ ]. Trk activation was blocked using CEP-4416 as previously reported (Mojsilovic-Petrovic et al., 2006) and biochemically confirmed here after acute (30 $\mathrm{min})$ or chronic $(6 \mathrm{~h})$ application of BDNF to cultures (supplemental Fig. 1, available at www.jneurosci.org as supplemental material).

Cell survival quantification. The total number of CM-DiI-labeled motor neurons was determined in a rectangular region of interest (RROI) defined by the Teflon walls ("east" and "west" boundaries) and successive scratches made by the pin comb ("north" and "south" boundaries). A RROI was chosen for study if the density of labeled cells was not prohibitively high and the precise location in the culture dish was noted so that we could return to the same RROI at a subsequent time point. For each culture dish, 4-5 RROIs were counted and summed. After an experimental manipulation, we determined the number of motor neurons in the specific RROIs that were previously counted. All results are expressed as the number of motor neurons in summed RROI after manipulation divided by the number of motor neurons at the initial time point $\times 100$ (“\% motor neuron survival”). In any given experiment, we averaged no less than 5 dishes/ condition. An example of the raw data that was used to generate "\% motor neuron survival" for Figure 1 is present in supplemental Table 1 (available at www.jneurosci.org as supplemental material).

Mixed spinal cord neuron cultures. To have sufficient material for biochemical determinations, we used mixed spinal cord neuron cultures in the surface biotinylation experiments.

E14-E16 embryonic rat spinal cord neurons were grown on confluent monolayers of cortical astrocytes, as described previously (Hu and Kalb, 2003).

Surface biotinylation. We performed cell surface biotinylation and immunoblotting as previously described (Zhou et al., 2008).

Immunocytochemistry, immunoprecipitation, and immunoblotting. Immunocytochemical studies were performed as previously described (Fryer et al., 2000). The following primary antibodies were used: antiMAP2 (1:100, Millipore) and SMI-32 and SMI-312 (1:1000, Covance). The anti-tau antibody was a generous gift from Dr. Virginia Lee (University of Pennsylvania, Philadelphia, PA). Alexa-conjugated secondary antibodies were from Invitrogen. Immunoprecipitation and immunoblotting were performed as previously described (Mojsilovic-Petrovic et al., 2006). The following primary antibodies were used: anti-phosphotyrosine (Millipore), anti-TrkB (Santa Cruz Biotechnology), antiphosphoAKT, anti-AKT, anti-phosphoMAPK, anti-MAPK, anti-human SOD (Cell Signaling Technology), and anti-actin (Sigma).

Statistics. Data are presented as mean \pm SD. ANOVA was used to compare groups, followed by post hoc analysis using Bonferroni adjustment for multiple comparisons procedure. The level of significance was set at $p<0.05$.
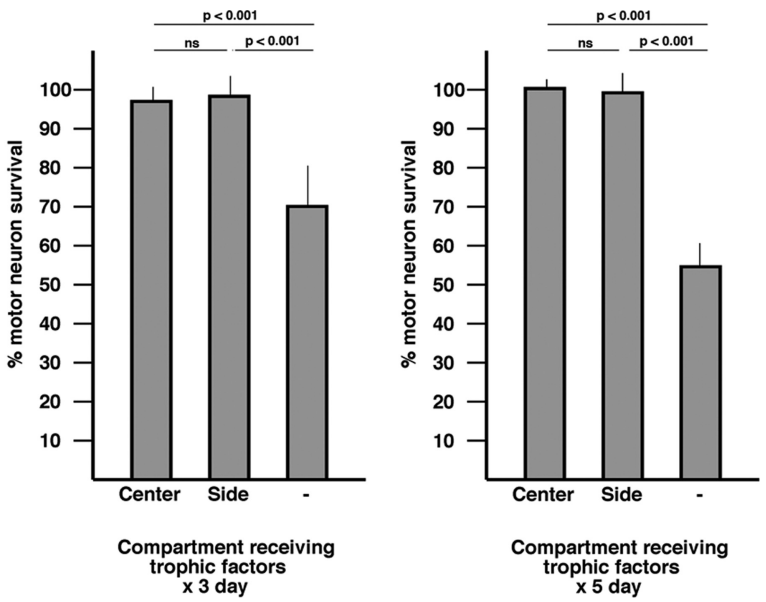

Figure 1. Trophic factors promote the survival of motor neurons when presented to the cell body or to axons/dendrites. Purified (the cell body or neurite compartments and cell survival was

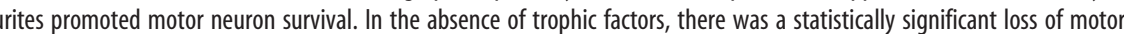

A

HSV-G37R-SOD

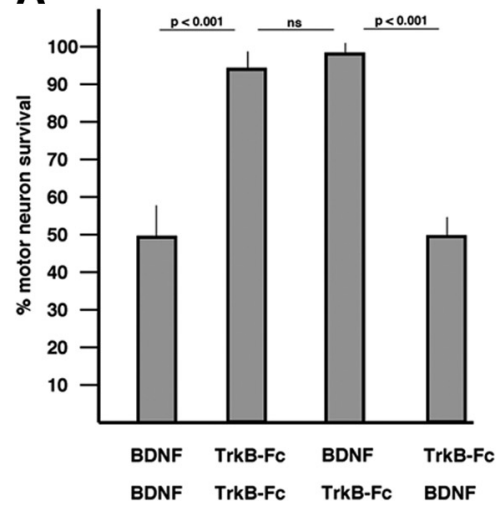

Figure 2. Motor neurons expressing mutant SOD die when stimulated by BDNF on axons or dendrites. Purified motor neurons were cultured in the cell body compartment of a chamber culture, infected with HSV-mutant SOD, and then grown in media with TrkB-Fc $(\boldsymbol{A})$ or with CEP4417 or vehicle in specified compartments $(\boldsymbol{B})$. In the experiment described by $\boldsymbol{A}$, motor neuron death occurred only when BDNF was present in the neurite compartment. In $\boldsymbol{B}$, motor neuron death was prevented by application of CEP-4416 to the neurite compartment.

\section{Results}

We found excellent long-term survival of purified motor neurons when grown in a cocktail of neurotrophic factors in the cell body and neurite compartments. Hoechst staining of the cell body or neurite compartments confirmed that the media in each compartment is isolated and that cells with nuclei exist exclusively in the cell body compartment (supplemental Fig. 2, available at www.jneurosci.org as supplemental material).

Within the first few days of culture, neurites begin to be visible in the neurite compartments and grow in number, length, and complexity over the subsequent 2 weeks (supplemental Fig. 3, available at www.jneurosci.org as supplemental material). Neurites expressed immunological markers for axons and dendrites.

Motor neurons with processes into the neurite compartments (labeled by neurite compartment CM-DiI) were stained with Hoechst, and this led us to estimate that $\sim 35 \%$ of motor neurons in the cell body compartment have processes in the neurite compartment. 
A

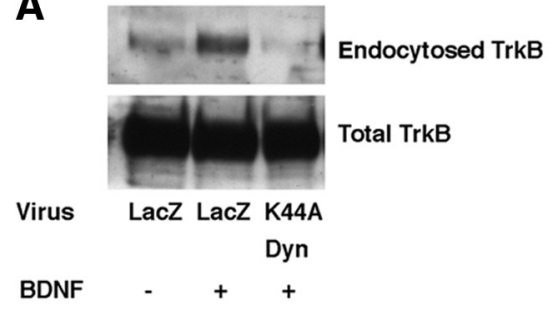

B

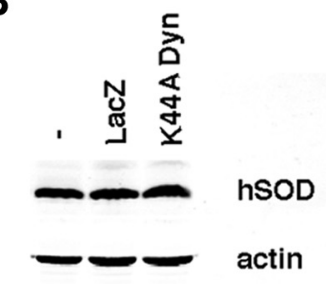

C

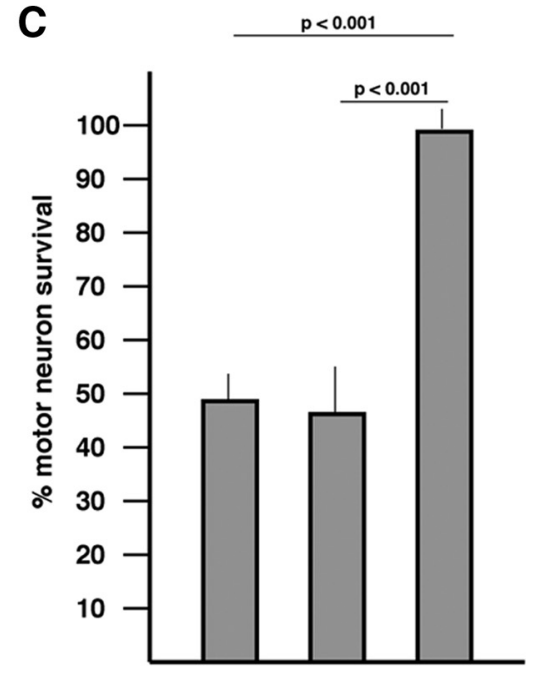

Virus in addition to

\section{LacZ}

K44A
Dyn.

Figure 3. Inhibition of endocytosis blocks the ability of BDNF to evoke vulnerability to mutant SOD toxicity. $A$, Mixed spinal cord cultures were infected with HSV engineered to express K44A dynamin or LacZ and then surface proteins were biotinylated. After stimulation with BDNF or vehicle, lysates were prepared and immunoprecipitated with streptavidin beads, and the pulldown material was immunoblotted for TrkB. The greater the signal, the more endocytosis has occurred. In cultures infected with LacZ, BDNF stimulates robust TrkB endocytosis, and this is completely blocked by expression of K44A dynamin. In $\boldsymbol{B}$, Western blot for human SOD in cultures infected with HSV-mutant SOD alone, or plus HSV-LacZ or HSV-K44A dynamin. The abundance of mutant SOD is the same in all conditions. In C, the effect of K44A dynamin on mutant SOD toxicity was examined. Purified motor neurons were cultured in the cell body compartment of a chamber culture, CM-Dil labeled, counted, and infected with HSV engineered to express mutant SOD plus HSV-LacZ or HSV-K44A dynamin. BDNF was present in all compartments throughout the experiment, and the percentage survival was determined $6 \mathrm{~d}$ later. Expression of K44A dynamin protected motor neurons from mutant SOD toxicity.

To examine spatial aspects of trophic factor survival signaling of motor neurons, we grew purified motor neurons in the cell body compartment of the compartment culture for 2 weeks in $\mathrm{NB}+$ CGC media labeled by CM-DiI, counted in specific RROIs and the values recorded. The next day, three experimental groups were generated: (1) NB + CGC media in the cell body compartment and NB in the neurite compartment, (2) NB + CGC media in the neurite compartment and NB in the cell body compartment, and (3) NB in both the cell body and neurite compartment. We then counted motor neurons in the previously noted RROIs at subsequent time points. For ease of presentation, we express results as percentage motor neuron survival (see Materials and Methods for details), but the absolute values are also provided (supplemental Table 1, available at www.jneurosci.org as supplemental material). At the $1 \mathrm{~d}$ time point, $99 \pm 2 \%$ of motor neurons survived when CGC was present in the cell body compartment, $101 \pm 1 \%$ of motor neurons survived when CGC was present in the neurite compartment, and $82 \pm 7 \%$ of motor neurons in the no CGC supplied group (group differences by ANOVA, $\left.F_{(2,21)}=18.29, p<0.0001\right)$. At the $3 \mathrm{~d}$ time point, the survival rates were $97 \pm 4 \%, 98 \pm 6 \%$, and $69 \pm 7 \%$ in the respective groups $\left(F_{(2,21)}=27.80, p<0.0001\right)$. At the $5 \mathrm{~d}$ time point, the survival rates were $100 \pm 2 \%, 99 \pm 4 \%$, and $54 \pm 6 \%$ in the respective groups $\left(F_{(2,21)}=225.1, p<0.0001\right)$ (Fig. 1; supplemental Table 1, available at www.jneurosci.org as supplemental material). These results indicate that provision of trophic factors to the motor neuron cell body or axon/dendrites strongly promotes survival.

In amyotrophic lateral sclerosis (ALS), motor neurons die, and the disease has been modeled in vivo and in vitro by expression in neurons of a mutant version of superoxide dismutase
(SOD). In previous in vitro work, we found that BDNF-TrkB signaling makes motor neurons susceptible to mutant SOD toxicity (Mojsilovic-Petrovic et al., 2006). This led us to ask whether the location of BDNF-TrkB signaling played a role in rendering cells vulnerable to insult. We began by preparing motor neuron cultures as above and infecting with HSV-G37Rmutant SOD at 14 DIV. The following day, all compartments were washed and the media replaced to generate the following experimental groups: (1) NB + CGC media including BDNF ("+BDNF") in all compartments, (2) NB + CGC media lacking BDNF and including TrkB-Fc (" $-\mathrm{BDNF} /$ + TrkB-Fc") in all compartments, (3) NB + CGC media +BDNF in the cell body compartment and NB + CGC media - BDNF/ + TrkB-Fc in the neurite compartment, and (4) NB + CGC media - BDNF/+TrkB-Fc in the cell body compartment and $\mathrm{NB}+$ CGC media +BDNF in the neurite compartment. Group differences were found with $\operatorname{ANOVA}\left(F_{(3,16)}=648.5, p<0.001\right)$, and post hoc tests showed that statistically significant motor neuron death was seen when BDNF was provided to all compartments in comparison with no BDNF in any compartment ( $49 \pm 5 \%$ vs $96 \pm 3 \%$, $p<$ 0.001) (Fig. $2 A$ ). In addition, motor neuron death was seen when BDNF was presented to the neurite compartment but not to the cell body compartment (51 $\pm 3 \%$ vs $98 \pm 2 \%, p<0.001)$ (Fig. $2 A)$. Thus, BDNF-TrkB signaling at axons/dendrites renders motor neurons susceptible to the toxic actions of mutant SOD.

We next examined the spatial aspects of BDNF signaling pharmacologically, using CEP-4416, a molecule that blocks activation of Trk receptors (Mojsilovic-Petrovic et al., 2006) (supplemental Fig. 1, available at www.jneurosci.org as supplemental material). Motor neuron cultures were prepared as above, infected with HSV-G37R-mutant SOD, and maintained with CGC + BDNF media. We compared survival in four experimental groups: (1) application of the TrkB antagonist CEP-4416 to the cell body and neurite compartments, (2) application of CEP-4416 to the cell body compartment and application of vehicle to the neurite compartments, (3) application of CEP-4416 to the neurite compartments and application of vehicle to the cell body compartment, and (4) application of vehicle only to the cell body and neurite compartments. By ANOVA, group differences were found $\left(F_{(3,25)}=350.7\right)$ (Fig. $\left.2 B\right)$. The post hoc analysis revealed that application of CEP-4416 in all compartments provided a statistically significant protection against mutant SOD toxicity in comparison with vehicle-only-treated cultures $(100 \pm 2 \%$ vs $50 \pm 5 \%$, $p<$ 0.001). In addition, CEP-4416 application to the neurite compartments was also neuroprotective, while its application to the cell body compartment conferred no protection ( $98 \pm 4 \%$ vs $42 \pm 2 \%, p<$ $0.001)$. These observations show that the "susceptibility signal" is generated by activation of TrkB in the axon/dendritic domain.

Upon binding BDNF, TrkB undergoes autophosphorylation, leading to activation of multiple signaling modules, and subsequently the ligand-receptor complex undergoes endocytosis. Since events triggered by cell-surface receptors can differ from 
those in which the ligand-receptor complex undergoes endocytosis (Zhang et al., 2000), we inquired whether blocking endocytosis influenced the ability of mutant SOD to kill motor neurons. To achieve this end, we engineered a HSV to express the K44A mutant of dynamin, a well described genetic tool for blocking endocytosis (Ye et al., 2003). To assess the efficacy of these tools, we biotinylated cell-surface proteins, stimulated endocytosis with BDNF, purified endocytosed proteins with avidin beads, and immunoblotted for TrkB. Upon addition of BDNF, there is a marked increase in the TrkB band indicative of robust ligandinduced internalization of the receptor in HSV-LacZ-infected neurons. HSV-K44A dynamin-infected cultures had no ligandinduced internalization of $\operatorname{TrkB}$ (Fig. $3 A$ ). The overall abundance of TrkB in these cultures was unaffected by the virus treatments. In addition, coexpression of HSV-LacZ or HSV-K44A dynamin with G37R-mutant SOD did not affect the expression level of SOD (Fig. 3B). These biochemical results demonstrate that in our tissue culture system, expression of $\mathrm{K} 44 \mathrm{~A}$ dynamin blocks BDNF-induced endocytosis of activated TrkB.

We next asked whether expression of K44A dynamin influenced mutant SOD-mediated motor neuron death. Motor neuron cultures were prepared as above and at 14 DIV infected with the following combinations of recombinant HSV: G37R mutant SOD, G37R mutant SOD + LacZ, or G37R mutant SOD + K44A dynamin. Motor neuron counts and ANOVA revealed differences between groups $\left(F_{(2,21)}=758.6, p<0.0001\right)$ (Fig. 3C). Post hoc analysis indicated a statistically significant promotion of motor neuron survival when coexpressing K44A dynamin with mutant SOD compared with mutant SOD alone (96 $\pm 3 \%$ vs $49 \pm 3$, $p<0.001$ ) or mutant SOD plus LacZ ( $96 \pm 3 \%$ vs $47 \pm 6 \%, p<$ 0.001 ). These results indicate that endocytosis (presumably of TrkB) in axons and/or dendrites of motor neurons is required for the generation of a signal that renders motor neurons vulnerable to the toxic actions of mutant SOD.

Since BDNF can activate protein synthesis in the axon/dendrite compartment (Takei et al., 2001; Schratt et al., 2004), we wondered whether the effects of active TrkB on axons/dendrites required local protein synthesis. In previous (noncompartment culture) studies, we found that exposing motor neurons to BDNF for 3-6 h was sufficient to render them susceptible to excitotoxic insult and that coadministration of $\mathrm{CHX}$ during the exposure to BDNF nullified the effect (Fryer et al., 2000). Exposure to CHX for $>12 \mathrm{~h}$ was toxic (data not shown). Here, motor neuron cultures were prepared as above and TrkB-Fc was added to all compartments before infecting cultures with HSV-G37R-SOD. Next, the neurite compartments of cultures were preincubated with 1 $\mu \mathrm{g} / \mathrm{ml} \mathrm{CHX}$ or vehicle for $1 \mathrm{~h}$ followed by stimulation of the neurite compartment with $50 \mathrm{ng} / \mathrm{ml} \mathrm{BDNF}$ for $6 \mathrm{~h}$. As a control, one group of cultures was treated with BDNF in the neurite compartment $6 \mathrm{~d}$ in the absence of any CHX treatment. Following the pulse with $\mathrm{BDNF}( \pm \mathrm{CHX})$, the neurite compartments were washed and maintained in CGC $-\mathrm{BDNF} /+$ TrkB-Fc media for $6 \mathrm{~d}$. We then counted the number of CM-DiI-labeled motor neurons, and the ANOVA indicated group differences $\left(F_{(2,27)}=\right.$ $38.78, p<0.0001)$. The post hoc analysis revealed a statistically significant protective effect of coadministration of cycloheximide during the BDNF when compared with motor neurons pulsed with BDNF (no CHX) (95 $\pm 3 \%$ vs $55 \pm 5 \%, p<0.001)$ or grown continuously with BDNF in the neurite compartment $(95 \pm 3 \%$ vs $51 \pm 2 \%, p<0.001$ ) (Fig. 4). These results show that protein synthesis in axons/dendrites is essential for the BDNF-TrkBevoked state of vulnerability to mutant SOD toxicity.

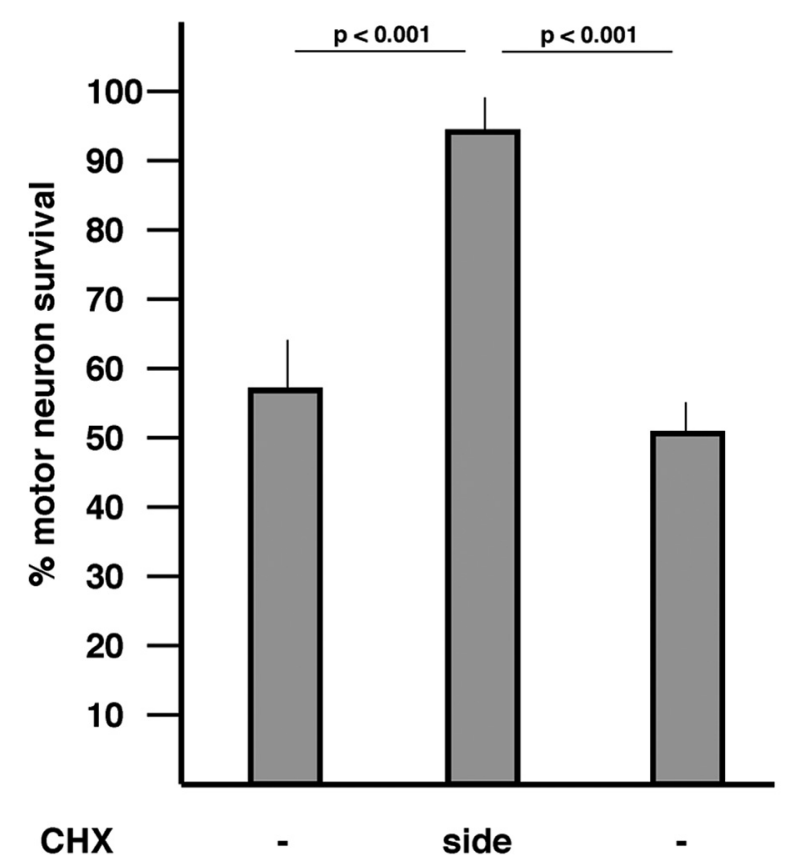

\section{BDNF, side pulse pulse continuous compartment}

Figure 4. Protein synthesis inhibition in axons/dendrites blocks the ability of BDNF to induce vulnerability to mutant SOD. Purified motor neurons were cultured in the cell body compartment of a chamber culture, CM-Dil labeled, counted, and then infected with HSV engineered to express mutant SOD for $24 \mathrm{~h}$. BDNF was introduced into neurite compartment for a $6 \mathrm{~h}$ pulse or for the next $6 \mathrm{~d}$. CHX or vehicle was present during the BDNF pulse. The percentage motor neuron death was determined $6 \mathrm{~d}$ later. Motor neuron death occurred when BDNF was continuously present in the neurite compartment. Motor neuron death also occurred if axons/dendrites were pulsed with BDNF, and this effect was blocked by coadministration of CHX.

\section{Discussion}

The biological effects of neurotrophin signaling vary as a function of the location on the neuron where the ligand binds its receptor. Here we demonstrate that motor neuron survival is promoted by trophic factor stimulation of either the cell body or axons/dendrites. Despite this equivalence, it is likely that trophic factor actions are different at these two neuronal locations. Only stimulation of the axon/dendrite domain with BDNF evokes a state of vulnerability of motor neurons to mutant SOD toxicity. It is noteworthy that muscle cells generate several trophic factors (including BDNF), and in ALS patients, the abundance of BDNF mRNA in muscle is particularly high early in the disease (Küst et al., 2002). This has been interpreted to mean that muscle cells increase BDNF expression in response to partial denervation early in the course of ALS to promote neuromuscular junction (NMJ) stability (Gonzalez et al., 1999). Our observations raise the possibility that activation of $\mathrm{TrkB}$ receptors on motor neuron axons might paradoxically exacerbate the pathophysiological process.

Exposure of axons or dendrites to BDNF induces translation of local mRNAs in a mammalian target of rapamycin-dependent manner (Schratt et al., 2004; Takei et al., 2004, 2009). This is likely to be relevant to BDNF-induced vulnerability of motor neurons to mutant SOD toxicity, since the effects of BDNF in the axon/ dendrite compartment are blocked by the protein synthesis inhibitor CHX. Considering that the pool of axonal mRNAs that could be translated in response to BDNF is deep, it is reasonable 
to hypothesize that BDNF induces the expression of a suite of proteins that together confer vulnerability to insult. Nevertheless one can identify specific proteins that are plausibly key players. For example, in cortical neuron culture, BDNF stimulates translation of the NADPH oxidase subunits gp91-phox (NOX2), p22phox, p47-phox, and p67-phox (Kim et al., 2002). Upon activation, NADPH oxidase reduces molecular oxygen to generate superoxide and other reactive oxygen species (ROS). Kim et al. (2002) show that the BDNF-stimulated increase in ROS leads to neuron death. In the G93A mutant SOD model of ALS, genetic deletion of NADPH oxidases (either NOX1 or NOX2) delays the onset and slows the progression of disease (Wu et al., 2006; Marden et al., 2007). Immunohistochemically, NOX2 was localized to microglia (Wu et al., 2006), although by in situ hybridization, NOX2 (as well as regulatory subunits p22phox, p47-phox, and p67-phox) is also present in motor neurons (Allen Brain Atlas, http://www.brain-map.org/). This example serves to illustrate that one mechanism by which BDNF could plausibly harm motor neurons is by stimulating the translation of proteins that increase oxidative stress.

The main finding in this report is that when BDNF activates TrkB on axons or dendrites, the complex undergoes endocytosis and stimulates the de novo synthesis of proteins that are important for mutant SOD toxicity of motor neurons. Given the increased scrutiny of events occurring in axons and the NMJ, we suggest a number of mechanisms that might, individually or collectively, be linked to the pathophysiology. Work in Caenorhabditis elegans has highlighted the functionally important role of axonal mRNA stability and translation under normal and pathological conditions (Yan et al., 2009). It is worth noting, however, that in vivo, TrkB is expressed on adult motor neuron cell bodies and dendrites, and BDNF is produced in the spinal cord parenchyma (Copray and Kernell, 2000) (Zhang and Huang, 2006). None of the results in this report exclude the possibility that the pathophysiologically significant processes are actually occurring in dendrites. Selective targeting of TrkB to axons or dendrites might allow us to address this issue. Ultimately, understanding the mechanism by which activation of TrkB on axons or dendrites renders motor neurons vulnerable to insult may permit new therapeutic approaches.

\section{References}

Campenot RB (1977) Local control of neurite development by nerve growth factor. Proc Natl Acad Sci U S A 74:4516-4519.

Copray S, Kernell D (2000) Neurotrophins and trk-receptors in adult rat spinal motoneurons: differences related to cell size but not to 'slow/fast' specialization. Neurosci Lett 289:217-220.

Fryer HJL, Wolf DH, Knox RJ, Strittmatter SM, Pennica D, O'Leary RM, Russell DS, Kalb RG (2000) Brain-derived neurotrophic factor induces excitotoxic sensitivity in cultured embryonic rat spinal motor neurons through activation of the phosphatidylinositol 3-kinase pathway. J Neurochem 74:582-595.

Gonzalez M, Ruggiero FP, Chang Q, Shi Y-J, Rich MM, Kraner S, BaliceGordon RJ (1999) Disruption of TrkB-mediated signaling induces disassembly of postsynaptic receptor clusters at the neuromuscular junction. Neuron 24:567-583.

Hamburger V (1958) Regression versus peripheral control of differentiation of motor hypoplasia. Am J Anat 102:365-409.

Hu P, Kalb RG (2003) BDNF heightens the sensitivity of motor neurons to excitotoxic insults through activation of TrkB. J Neurochem 84: $1421-1430$.
Ishikawa Y, Ikeuchi T, Hatanaka H (2000) Brain-derived neurotrophic factor accelerates nitric oxide donor-induced apoptosis of cultured cortical neurons. J Neurochem 75:494-502.

Kim HJ, Hwang JJ, Behrens MM, Snider BJ, Choi DW, Koh JY (2003) TrkB mediates BDNF-induced potentiation of neuronal necrosis in cortical culture. Neurobiol Dis 14:110-119.

Kim SH, Won SJ, Sohn S, Kwon HJ, Lee JY, Park JH, Gwag BJ (2002) Brainderived neurotrophic factor can act as a pronecrotic factor through transcriptional and translational activation of NADPH oxidase. J Cell Biol 159:821-831.

Koh JY, Gwag BJ, Lobner D, Choi DW (1995) Potentiated necrosis of cultured cortical neurons by neurotrophins. Science 268:573-575.

Kuruvilla R, Ye H, Ginty DD (2000) Spatially and functionally distinct roles of the PI3-K effector pathway during NGF signaling in sympathetic neurons. Neuron 27:499-512.

Küst BM, Copray JC, Brouwer N, Troost D, Boddeke HW (2002) Elevated levels of neurotrophins in human biceps brachii tissue of amyotrophic lateral sclerosis. Exp Neurol 177:419-427.

Marden JJ, Harraz MM, Williams AJ, Nelson K, Luo M, Paulson H, Engelhardt JF (2007) Redox modifier genes in amyotrophic lateral sclerosis in mice. J Clin Invest 117:2913-2919.

Mojsilovic-Petrovic J, Jeong GB, Crocker A, Arneja A, David S, Russell DS, Kalb RG (2006) Protecting motor neurons from toxic insult by antagonism of adenosine A2a and Trk receptors. J Neurosci 26:9250-9263.

Schratt GM, Nigh EA, Chen WG, Hu L, Greenberg ME (2004) BDNF regulates the translation of a select group of mRNAs by a mammalian target of rapamycin-phosphatidylinositol 3-kinase-dependent pathway during neuronal development. J Neurosci 24:7366-7377.

Segal RA (2003) Selectivity in neurotrophin signaling: theme and variations. Annu Rev Neurosci 26:299-330.

Takei N, Kawamura M, Hara K, Yonezawa K, Nawa H (2001) Brain-derived neurotrophic factor enhances neuronal translation by activating multiple initiation processes: comparison with the effects of insulin. J Biol Chem 276:42818-42825.

Takei N, Inamura N, Kawamura M, Namba H, Hara K, Yonezawa K, Nawa H (2004) Brain-derived neurotrophic factor induces mammalian target of rapamycin-dependent local activation of translation machinery and protein synthesis in neuronal dendrites. J Neurosci 24:9760-9769.

Takei N, Kawamura M, Ishizuka Y, Kakiya N, Inamura N, Namba H, Nawa H (2009) Brain-derived neurotrophic factor enhances the basal rate of protein synthesis by increasing active eukaryotic elongation factor 2 levels and promoting translation elongation in cortical neurons. J Biol Chem 284:26340-26348.

Watson FL, Heerssen HM, Bhattacharyya A, Klesse L, Lin MZ, Segal RA (2001) Neurotrophins use the Erk5 pathway to mediate a retrograde survival response. Nat Neurosci 4:981-988.

Wu DC, Ré DB, Nagai M, Ischiropoulos H, Przedborski S (2006) The inflammatory NADPH oxidase enzyme modulates motor neuron degeneration in amyotrophic lateral sclerosis mice. Proc Natl Acad Sci U S A 103:12132-12137.

Yan D, Wu Z, Chisholm AD, Jin Y (2009) The DLK-1 kinase promotes mRNA stability and local translation in C. elegans synapses and axon regeneration. Cell 138:1005-1018.

Ye H, Kuruvilla R, Zweifel LS, Ginty DD (2003) Evidence in support of signaling endosome-based retrograde survival of sympathetic neurons. Neuron 39:57-68.

Zhang J, Huang EJ (2006) Dynamic expression of neurotrophic factor receptors in postnatal spinal motoneurons and in mouse model of ALS. J Neurobiol 66:882-895.

Zhang Y, Moheban DB, Conway BR, Bhattacharyya A, Segal RA (2000) Cell surface Trk receptors mediate NGF-induced survival while internalized receptors regulate NGF-induced differentiation. J Neurosci 20:56715678.

Zhou W, Zhang L, Guoxiang X, Mojsilovic-Petrovic J, Takamaya K, Sattler R, Huganir R, Kalb R (2008) GluR1 controls dendrite growth through its binding partner, SAP97. J Neurosci 28:10220-10233. 